\title{
Preface
}

\section{Perioperative Management of the Thoracic Patient Continues to Evolve}

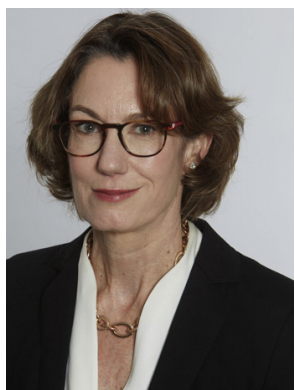

Virginia R. Litle, MD

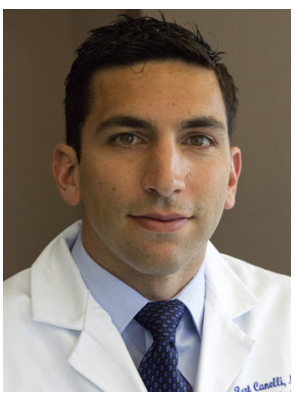

Robert J. Canelli, MD

Editors

We are excited to bring you this focused issue for the Thoracic Surgery Clinics on "Perioperative Management of the Thoracic Patient." In contrast to other surgical specialties, general thoracic surgery in particular requires deliberate coordination between all members of the operative team. To optimize patient safety and operative efficiency, anesthesiologists and surgeons must communicate not only intraoperatively but also preoperatively as well. General thoracic surgeons and anesthesiologists need to know how to take an evidence-based approach to manage perioperative problems. Lung resections and esophagectomies are fraught with the overriding risks of intraoperative and postoperative complications. Mitigating risk starts with a shared decision-making conversation between patient and surgeon, once the patient elects to pursue operative therapy for their thoracic malignancy, thymic lesion, or benign esophageal disease. From the preoperative gateway, down the intraoperative path, and through discharge, the patient will travel safely with guidance from the expert contributions presented in the following pages.
Matheos and colleagues lead us off with an update on the preoperative evaluation and include an algorithm for cardiopulmonary testing. The preoperative management of thoracic patients has evolved beyond simply pulmonary function tests and the as-needed stress echocardiogram. Dezube and colleagues outline a prehabilitation program that calculates a patient's frailty index and considers physiologic versus chronologic age. Prehabilitation will optimize patients before surgery, so that they may sprint down the operative road without stumbling. Unlike Prehabilitation, which remains fairly nascent in our field, enhanced recovery after surgery (ERAS) programs have become increasingly routine in our field, perhaps because they are less resource intensive. Haywood and coauthors take us through the more immediate preoperative nutritional guidelines to the intraoperative anesthetic suggestions with the ultimate goal of reducing the patient's postoperative pain and return to homeostasis.

Intraoperative management is a component of ERAS; however, Shoni and Rodriguez discuss patient-specific ventilator management strategies and one-lung ventilation complications. For the 
surgeons, the Pittsburgh team of Witek and colleagues offers decision trees for operative approaches, including minimally invasive versus open and choice of type of esophagectomy depending on details of patient disease. Geraci, with senior author Cerfolio, applies their extensive robotic experience to cover both surgical and anesthetic concerns for mediastinal, pulmonary, and esophageal operations. Intraoperative guidance on how to reduce postoperative complications like air leaks and anastomotic leaks is well summarized by surgical colleagues in their contributions; however, when such complications arise, Clark and colleagues and Yeung offer management options.

We have asked our contributors to delineate the steps for optimal perioperative management of the complex thoracic patient. The mutual goal of the anesthesiologists and surgeons managing patients with lung or esophagus cancer in particular is patient safety. Thank you to our contributors. We hope you will enjoy this issue!

Virginia R. Litle, MD Division of Thoracic Surgery Department of Surgery 88 East Newton Street Collamore Building, Suite 7380 Boston, MA 02118, USA

Robert J. Canelli, MD Boston Medical Center Department of Anesthesiology 750 Albany Street, Suite 2R Boston, MA 02118, USA

E-mail addresses:

Virginia.litle@bmc.org (V.R. Litle) Robert.canellibmc.org (R.J. Canelli) 